\title{
Coarse to Fine Segmentation of Stargardt Rings using an Expert Guided Dual Ellipse Model
}

\section{${ }^{1}$ Noah Lee, ${ }^{1}$ Andrew F. Laine, and ${ }^{2} R$. Theodore Smith}

${ }^{1}$ Columbia University, Department of Biomedical Engineering, New York, NY, U.S.A. ${ }^{2}$ Columbia University, Department of Ophthalmology, New York, NY, U.S.A.

\section{INTRODUCTION}

Computer aided diagnosis (CAD) in the medical image domain requires adaptive knowledge-based models to handle uncertainty, ambiguity, and noise.

We propose an expert guided coupled dual ellipse model in a coarse to fine energy minimization framework.

Quantitative evaluations on synthetic and real data sets show the performance of our framework. Experimental results demonstrate that our framework performs well with an area under the ROC curve of 0.93 .

\section{MATERIALS \& METHODOLOGY}

2.1 Input Data

Retinal fundus images showing bright Stargardt ring structures. The bright ring is an abnormal accumulation of lipofuscin surrounding the dark bright ring is an abnormal accumulation of lipofuscin surrounding the dark
macular center region and can manifest itself in a variety of disease macular cente.

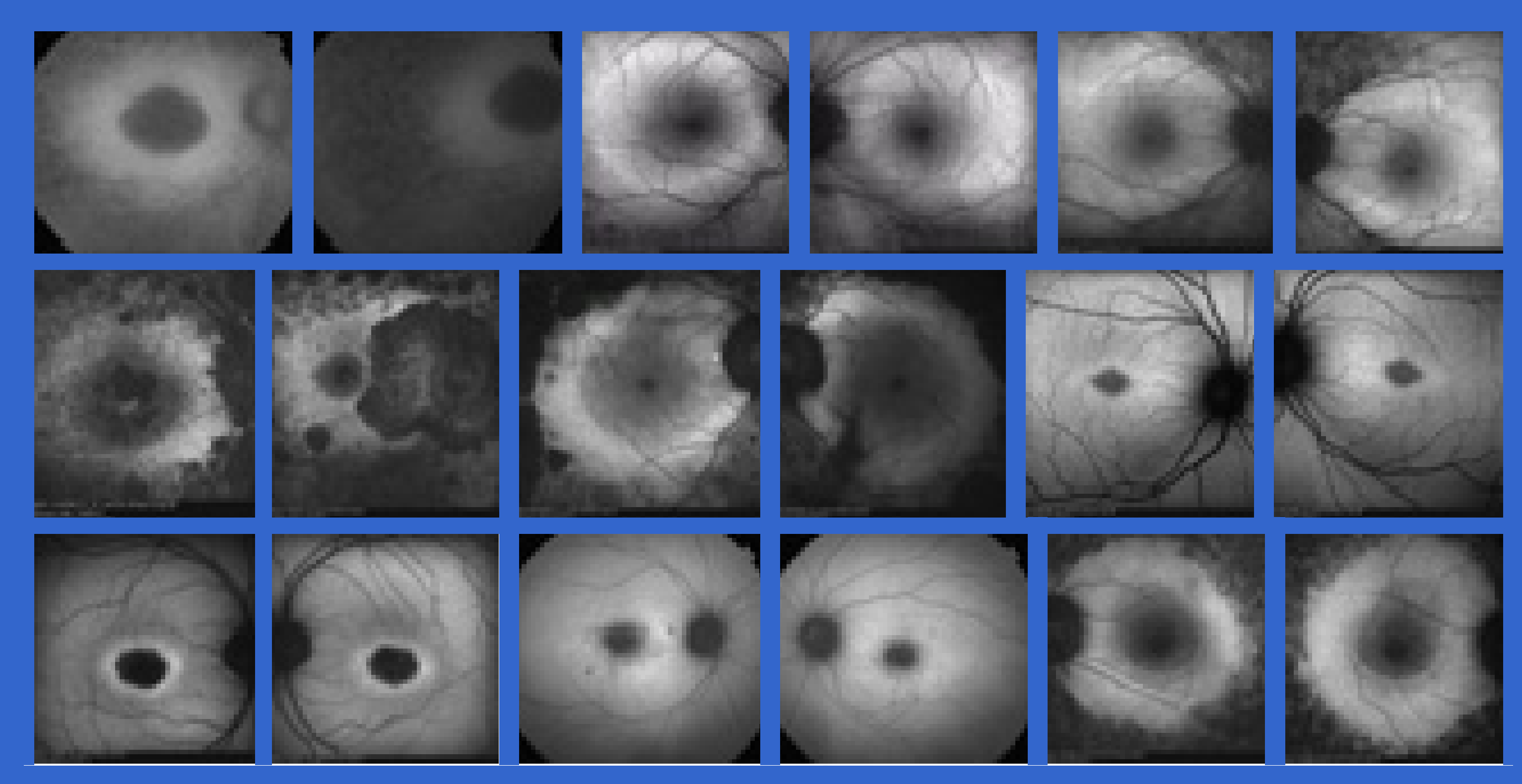

Our approach follows the spirit of multi-stage decomposition when analyzing a signal. Prior information from the coarse level guides as a modeling constraint the finer successive detail level when facing data ambiguities.

$$
E\left(\Theta, \phi, \phi_{\text {prior }}\right)=\underbrace{E_{1}(\Theta)}_{\text {coarse level }}+\underbrace{E_{2}\left(\phi, \phi_{\text {prior }}\right)}_{\text {fine level }}
$$

\subsection{The Coupled Dual Ellipse Model}

We use Euclidean parametric ellipse models to model a closed ring topology representing the Stargardt ring structure yet constraint in the space of elliptical shapes.

The model is flexible in a sense that the expert is able to partially provide model information within the parameter space for minimal expert intervention .

\subsection{Coarse Level Model Minimization}

The expert provides two seed locations placing a prior constraint on the approximate location of the inner and outer ellipse model. We evolve the coupled parametric model towards each other by minimizing for the ring area.

$$
\underset{a_{i}, b_{i}, x_{i}, y_{i}, \rho_{i}}{\operatorname{argmin}} \int_{\Theta_{\text {out }}} d \Theta_{o u t}-\int_{\Theta_{i n}} d \Theta_{i n}
$$

Coarse level minimization of the ring happens through optimization for three energy terms.

\section{MATERIALS \& METHODOLOGY}

The energy functional for the coarse level:

$$
E_{1}\left(f, \Theta_{\text {in }}, \Theta_{\text {out }}\right)=E_{\text {data }}+E_{\text {shape }}+\operatorname{area}(D)
$$

We drop an explicit smoothness constraint due to the implicit representation of the coupled dual ellipse model. The data term is a variant of the Mumford -Shah functional minimizing the within class variance of the intensity distribution. The shape term acts as a deflating force.

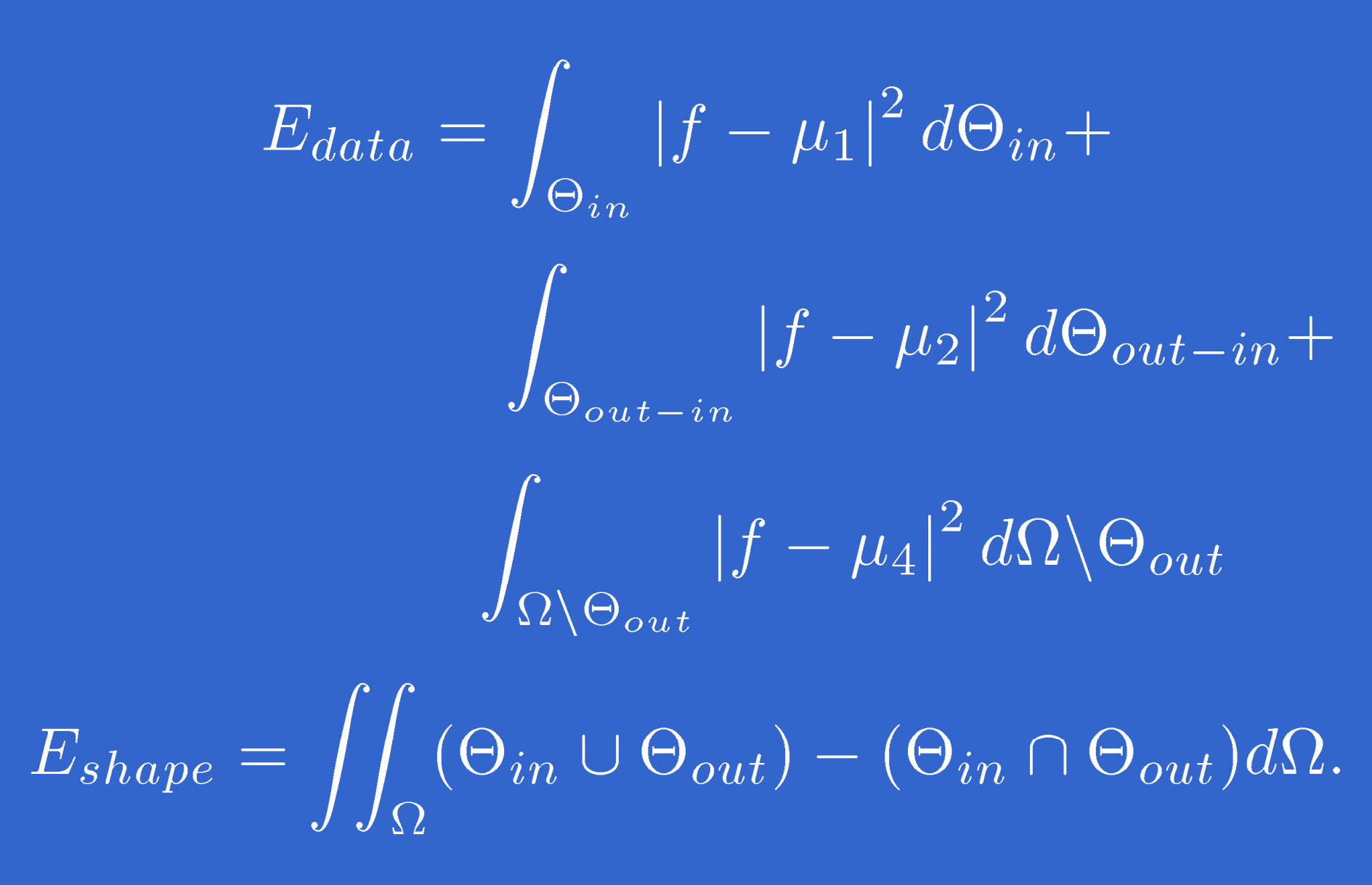

2.4 Fine Level Model Minimization

To model the fine detail level we transform the coarse level subspace constraint into an implicit representation suitable for shape matching to constrain the fine detail model. Optimization happens in the level set framework proposed by Osher and Sethian [1].

$$
E_{2}\left(f, \phi, \phi_{\text {prior }}\right)=\alpha \cdot E_{\text {data }}(\phi)+\beta \cdot E_{\text {shape }}\left(\phi_{\text {prior }}\right)
$$

In the context of the joint minimization problem the prior term is borrowed invisibly from the coarse level model through transformation to a higher dimensional embedding function.

For the fine level model we minimize an energy functional composed of a data term and expert guided shape constraint.

$$
\begin{aligned}
E_{\text {data }}(\phi)= & \int_{\Omega}\left(f-\mu_{\text {ring }}\right)^{2} H_{\epsilon}(\phi) d \Omega+ \\
& \int_{\Omega}\left(f-\mu_{b g}\right)^{2}\left(1-H_{\epsilon}(\phi)\right) d \Omega \\
E_{\text {shape }}\left(\phi, \phi_{\text {prior }}\right)= & \int_{\Omega}\left(\phi-\phi_{\text {prior }}\right)^{2} z \\
z & =\frac{1}{2}\left(h(\phi)+h\left(\phi_{\text {prior }}\right)\right) d \Omega .
\end{aligned}
$$

We keep the intensity mean values within the intensity mean of the subspace constraint and its background mean. The shape terms is modeled through a shape distance in $L_{2}$ with a normalization factor as

\begin{tabular}{|c|c|c|c|c|}
\hline $\begin{array}{c}\text { ROC } \\
\text { Statistic }\end{array}$ & $\begin{array}{l}\text { Noise } \\
\text { Coarse }\end{array}$ & $\begin{array}{c}\text { Noise } \\
\text { Fine }\end{array}$ & $\begin{array}{l}\text { Real } \\
\text { Coarse }\end{array}$ & $\begin{array}{l}\text { Real } \\
\text { Fine }\end{array}$ \\
\hline$\mu_{\text {sensitivity }}$ & .888 & .830 & .819 & .787 \\
\hline$\mu_{\text {specificity }}$ & .968 & .968 & .919 & .962 \\
\hline$\sigma_{\text {sensitivity }}$ & .016 & . 091 & .093. & . 076 \\
\hline$\sigma_{\text {specificity }}$ & .011 & .063 & .064 & .043 \\
\hline$A z_{R O C}$ & .885 & .938 & 903 & 930 \\
\hline
\end{tabular}
proposed in Cremers et al [2].

\section{RESULTS AND DISCUSSION}

We evaluate our method on synthetic and 10 real datasets to measure noise sensitivity and the ROC performance.

We measure the performance of the coarse and fine segmentation step and obtain the following ROC statistics for the synthetic and the retinal Stargardt test cases.
When observing the $A z$ value the performance of the coarse and the fine segmentation results are comparable with a slightly better performance of the fine segmentation of $2.7 \%$. We observer similar performance on the synthetic noise dataset. For the real data experiment the coarse results show slightly higher variations of the mean sensitivity and specificity.

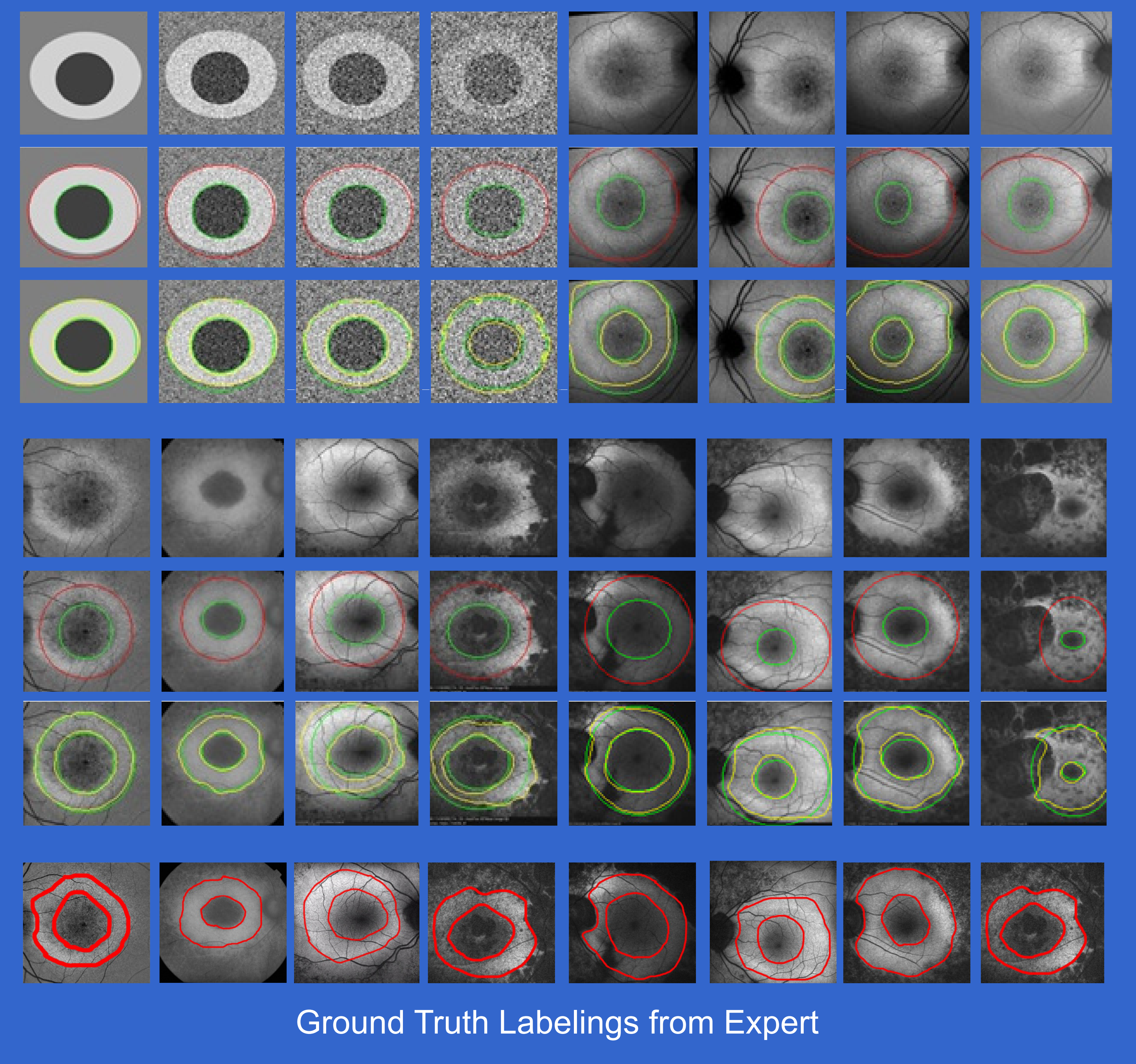

(Top Left) Segmentation results on 4 synthetic images using Gaussian noise of $25 \%, 50 \%$, and $100 \%$. The middle row shows fitted coupled dual ellipse model in red/green. Bottom row shows the final segmentation result.

(Top Right, Bottom Left/Right) Qualitative segmentation results on 12 retina Stargardt images comprised of different levels of segmentation complexity. The red/green contours denote the shape prior computed in Section 2.3 . The yellow/green contours show the final segmentation result.

\section{ACKNOWLEDGEMENTS}

This work was supported by the Imaging and Visualization Department at Siemens Corporate Research.

\section{SUMMARY \& CONCLUSIONS}

We have presented a coarse to fine minimization framework using a coupled dual ellipse model to form a subspace constraint that is used as a shape prior for fine model refinement.

Our experiments show promising results. Future work is devoted toward extension of our framework to deal with other ring like disease phenotypes.

\section{REFERENCES}

S. J. Osher and J. A. Sethian: Fronts propagation with
Jacobi formulations. J. of Comp. Phys., 79:12, 1988.

D. Cremerrs, S. Soatti: A pseuddo-distance for shape prior
Ceomentric and Level Set Methods Nice, $169-176,2003$. 